\title{
Anomalies and discrete chiral symmetries
}

\author{
Michael Creutz* ${ }^{\dagger}$ \\ Brookhaven National Laboratory and Universität Mainz \\ E-mail: creutz@bnl.gov
}

\begin{abstract}
The quantum anomaly that breaks the U(1) axial symmetry of massless multi-flavored QCD leaves behind a discrete flavor-singlet chiral invariance. With massive quarks, this residual symmetry has a close connection with the strong $\mathrm{CP}$-violating parameter theta. One result is that if the lightest quarks are degenerate, then a first order transition will occur when theta passes through pi. The resulting framework helps clarify when the rooting prescription for extrapolating in the number of flavors is valid.
\end{abstract}

International Workshop on QCD Green's Functions, Confinement and Phenomenology

September 7-11, 2009

ECT Trento, Italy

*Speaker.

$\dagger$ I am grateful to the Alexander von Humboldt Foundation for support for my ongoing visit to the University of Mainz. This manuscript has been authored under contract number DE-AC02-98CH10886 with the U.S. Department of Energy. Accordingly, the U.S. Government retains a non-exclusive, royalty-free license to publish or reproduce the published form of this contribution, or allow others to do so, for U.S. Government purposes. 


\section{Introduction}

As is well known to this community, chiral symmetry plays a crucial role in our understanding of QCD. But, of course, chiral symmetry is broken, and there are three sources of this breaking. First is the spontaneous breaking giving rise to an expectation value for the chiral condensate; i.e. $\langle\bar{\psi} \psi\rangle \neq 0$. This is invoked to explain the lightness of pions relative to other hadrons. Second, we have the implicit breaking of the flavor-singlet axial $U(1)$ symmetry by the anomaly. This explains why the $\eta^{\prime}$ meson is not so light in comparison with the pions. And finally we have the explicit breaking of chiral symmetry by the quark masses. This means that the pions, while light, are not exactly massless. In this talk I will discuss some of the rather rich physics that arises from the interplay of these three effects.

The breaking of the classical $U(1)$ axial symmetry is tied to the possibility of introducing into massive QCD a CP violating parameter, usually called $\Theta$. For a recent review of this quantity, see Ref. [1]. One of my goals here is to provide an intuitive and qualitative picture of the $\Theta$ parameter in meson physics. This picture has evolved over many years. The possibility of the spontaneous CP violation occurring at $\Theta=\pi$ is tied to what is known as Dashen's phenomenon [2], first noted even before the days of QCD. In the mid 1970's, 't Hooft [3] elucidated the underlying connection between the chiral anomaly and the topology of gauge fields. Later Witten [4] used large gauge group ideas to discuss the behavior at $\Theta=\pi$ in terms of effective Lagrangians. Ref. [5] lists a few of the early studies of the effects of $\Theta$ on effective Lagrangians. The topic continues to appear in various contexts; for example, Ref. [6] contains a different approach to understanding the transition at $\Theta=\pi$ in the framework of the two-flavor Nambu Jona-Lasinio model.

I became interested in these issues while trying to understand the difficulties with formulating chiral symmetry on the lattice. Much of the picture presented here is implicit in my 1995 paper on quark masses [7]. Since then the topic has become highly controversial, with the realization of ambiguities precluding a vanishing up quark mass as a solution to the strong $\mathrm{CP}$ problem [8] and the appearance of an inconsistency with one of the popular algorithms in lattice gauge theory [9]. Despite the controversies, both results are immediate consequences of the interplay of the anomaly and chiral symmetry. The fact that these issues remain so disputed drives me to return to them here. Portions of this discussion appear in more detail in Ref. [10].

A crucial issue is that the axial anomaly in $N_{f}$ flavor massless QCD leaves behind a residual $Z_{N_{f}}$ flavor-singlet chiral symmetry. This is closely tied to gauge-field topology and the QCD theta parameter. As a consequence I will show that, with degenerate quarks carrying a small non-zero mass, there must appear a first order transition at $\Theta=\pi$. For two flavors this transition studied in Refs. [7, 11, 12]. This result in turn has several further consequences. First, the sign of the quark mass is relevant for an odd number of flavors. This is not seen in perturbation theory, giving a simple example where perturbation theory does not provide a complete description of a field theory. Second, going down to one flavor, chiral symmetry no longer provides an additive protection for a small fermion mass. And third, the nontrivial dependence on the number of flavors can in some cases invalidate the rooting prescription often used to extrapolate between different flavor contents in lattice simulations. It is the latter two points which have been extremely controversial. 


\section{Assumptions}

For the purposes of this talk I make a few minimal assumptions. Considering QCD with $N_{f}$ light quarks, I assume this field theory exists and confines in the usual way. I assume that spontaneous chiral symmetry breaking occurs in the massless theory with $\langle\bar{\psi} \psi\rangle \neq 0$. When masses are considered, I consider that the usual chiral perturbation theory in momenta and masses makes sense. I also assume that the anomaly gives the $\eta^{\prime}$ a mass even when the quark masses vanish. I further consider $N_{f}$ small enough to avoid any possible conformal phases.

I frame the discussion in continuum language, but I imagine some non-perturbative regulator is in place to control divergences. Of course for me this would be the lattice, but I need not be more specific here. I assume this regulator has brought us close to the continuum theory, i.e. any momentum space cutoff should be much larger than $\Lambda_{Q C D}$, the natural scale of the strong interactions. For a lattice approach, the lattice spacing $a$ is considered as much smaller than $1 / \Lambda_{Q C D}$.

I consider the effective potential $V$ for various meson fields. This represents the resulting vacuum energy density for a given field expectation. Such can be derived formally via a Legendre transformation in the standard way. Here I will ignore convexity issues associated with the phase separation that will occur when a field is constrained to be in a naively concave region. A more precise treatment would be in terms of the phase transitions that occur with global minimum changes. Instead I proceed with the generally familiar language of symmetry breaking in terms of multiple minima in the effective potential. For simplicity I concentrate on $N_{f}$ degenerate quarks. To start I will also take $N_{f}$ even; this is because of some interesting subtleties with an odd number of flavors that I will get to later in the talk.

I will be considering a variety of composite fields. Because these are generally singular products of fields at the same space time point, I assume that our unspecified regulator has some way of handling this. The particular fields I will work with are

$$
\begin{gathered}
\sigma \sim \bar{\psi} \psi \\
\pi_{\alpha} \sim i \bar{\psi} \lambda_{\alpha} \gamma_{5} \psi \\
\eta^{\prime} \sim i \bar{\psi} \gamma_{5} \psi .
\end{gathered}
$$

Here $\lambda_{\alpha}$ represents the generalized Gell-Mann matrices generating the flavor group $S U\left(N_{f}\right)$.

\section{Spontaneous chiral symmetry breaking}

Spontaneous breaking of chiral symmetry is a crucial part of our understanding of the strong interactions. It is usually discussed in terms of a double well structure for the effective potential considered as a function of the field $\sigma \sim \bar{\psi} \psi$. The vacuum selects one of these minima giving an expectation value to the sigma field, $\langle\sigma\rangle=v \neq 0$. When the mass vanishes it is a convention whether one takes the positive or the negative minimum.

With multiple flavors the vacuum is continuously degenerate, with the non-singlet pseudoscalars being Goldstone bosons. This is associated with a symmetry under flavored chiral rotations of the quark fields

$$
\begin{aligned}
\psi & \rightarrow e^{i \phi \gamma_{5} \lambda^{\alpha} / 2} \psi \\
\bar{\psi} & \rightarrow \bar{\psi} e^{i \phi \gamma_{5} \lambda^{\alpha} / 2}
\end{aligned}
$$




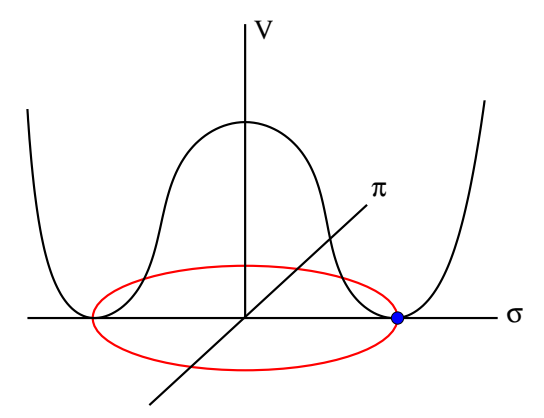

Figure 1: Spontaneous chiral symmetry breaking is represented by a double well effective potential with the vacuum settling into a non-trivial minimum. Chiral symmetry is broken by the selection of a specific value for the quark condensate. The flavor non-singlet pseudo-scalar mesons are Goldstone bosons corresponding to flat directions in the effective potential.

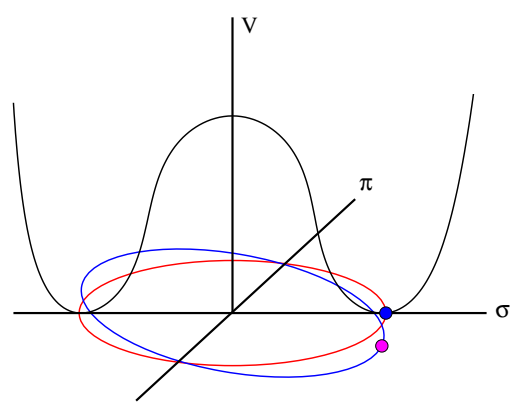

Figure 2: A small quark mass tilts the effective potential, selecting one direction for the true vacuum and giving the Goldstone bosons a mass.

There is one such symmetry for each generator of the flavor group $S U\left(N_{f}\right)$. For example, with two flavors this symmetry mixes the $\sigma$ and $\pi$ fields

$$
\begin{gathered}
\sigma \rightarrow \cos (\phi) \sigma+\sin (\phi) \pi^{\alpha} \\
\pi^{\alpha} \rightarrow \cos (\phi) \pi^{\alpha}-\sin (\phi) \sigma .
\end{gathered}
$$

The minimum of the potential has $N_{f}^{2}-1$ "flat" directions. This standard scenario is illustrated in Fig. 1.

If we now consider a small quark mass, this will select one vacuum as unique. Physically, a mass term represented by $V \rightarrow V-m \sigma$ tilts the effective potential downward in a specific direction, as illustrated in Fig. 2. In the process the Goldstone bosons acquire a mass proportional to the square root of the quark mass.

\section{The chiral anomaly}

It is the chiral anomaly that gives the flavor-singlet $\eta^{\prime}$ a mass even if the quark mass vanishes. This mass is of order the scale of the strong interactions, $m_{\eta^{\prime}}=O\left(\Lambda_{Q C D}\right)$, and does not go to zero when the quark mass does. In terms of the effective potential, $V\left(\sigma, \eta^{\prime}\right)$ is not symmetric under the rotation

$$
\begin{aligned}
\sigma & \rightarrow \sigma \cos (\phi)+\eta^{\prime} \sin (\phi) \\
\eta^{\prime} & \rightarrow \eta^{\prime} \cos (\phi)-\sigma \sin (\phi) .
\end{aligned}
$$


If we expand the effective potential near the vacuum state $\sigma \sim v$ and $\eta^{\prime} \sim 0$ we should expect a form like

$$
V\left(\sigma, \eta^{\prime}\right)=m_{\sigma}^{2}(\sigma-v)^{2}+m_{\eta^{\prime}}^{2} \eta^{\prime 2}+O\left((\sigma-v)^{3}, \eta^{\prime 4}\right)
$$

with both masses being of order $\Lambda_{Q C D}$.

In quark language, the above rotation mixing the $\sigma$ and $\eta^{\prime}$ fields is associated with the classical symmetry of the naive action under

$$
\begin{gathered}
\psi \rightarrow e^{i \phi \gamma_{5} / 2} \psi \\
\bar{\psi} \rightarrow \bar{\psi} e^{i \phi \gamma_{5} / 2} .
\end{gathered}
$$

This symmetry is "anomalous" in the sense that it must be broken by any valid regulator. The $\eta^{\prime}$ mass is a remnant of this breaking that survives as the regulator is removed.

Fujikawa [13] has presented a rather elegant way to see how the anomaly arises. The above variable change alters the fermion measure

$$
d \psi \rightarrow\left|e^{-i \phi \gamma_{5} / 2}\right| d \psi=e^{-i \phi \operatorname{Tr} \gamma_{5} / 2} d \psi
$$

Now naively $\gamma_{5}$ is a traceless matrix, and one might conclude that this change in the measure is harmless. Fujikawa pointed out that this does not apply in the regulated theory. For example one might define the trace of $\gamma_{5}$ as

$$
\lim _{\Lambda \rightarrow \infty} \operatorname{Tr}\left(\gamma_{5} e^{-D^{2} / \Lambda^{2}}\right) \neq 0
$$

where $D$ is the kinetic part of the Dirac action $\bar{\psi}(D+m) \psi$. In the usual continuum analysis this satisfies $D^{\dagger}=-D$ and anti-commutes with gamma five, $\left[D, \gamma_{5}\right]_{+}=0$. Thus motivated, we can use the eigenstates of $D$

$$
D\left|\psi_{i}\right\rangle=\lambda_{i}\left|\psi_{i}\right\rangle
$$

to define the trace

$$
\operatorname{Tr} \gamma_{5}=\sum_{i}\left\langle\psi_{i}\left|\gamma_{5}\right| \psi_{i}\right\rangle
$$

At this point we bring in the index theorem; this states that if the gauge field has non-trivial winding $v, D$ will have at least $v$ zero modes $D\left|\psi_{i}\right\rangle=0$. These modes are chiral: $\gamma_{5}\left|\psi_{i}\right\rangle= \pm\left|\psi_{i}\right\rangle$ and the counting is such that $v=n_{+}-n_{-}$. Thus the zero modes contribute $v$ to the trace of $\gamma_{5}$.

Now the non-zero eigenmodes all occur in complex conjugate pairs. If we have $D|\psi\rangle=$ $\lambda|\psi\rangle$, then $D \gamma_{5}|\psi\rangle=-\lambda \gamma_{5}|\psi\rangle=\lambda^{*} \gamma_{5}|\psi\rangle$. As $D$ is anti-hermitian, $|\psi\rangle$ and $\left|\gamma_{5} \psi\right\rangle$ are orthogonal whenever $\lambda \neq 0$. As a consequence, the space spanned by $|\psi\rangle$ and $\left|\gamma_{5} \psi\right\rangle$ gives no contribution to $\operatorname{Tr} \gamma_{5}$. We are led to the remarkable conclusion that only the zero modes count in calculating the above trace. Thus we have

$$
\operatorname{Tr} \gamma_{5}=\sum_{i}\left\langle\psi_{i}\left|\gamma_{5}\right| \psi_{i}\right\rangle=v
$$

which does not vanish when the topology is non-trivial.

So where did the opposite chirality states go? In continuum language, they are "lost at infinity" in the sense that they have been driven "above the cutoff." On the lattice there are no infinities; so things are a bit more subtle. With the overlap operator [14], all eigenvalues lie on a circle in the complex plane, and corresponding to every zero mode is a corresponding mode of opposite chirality 


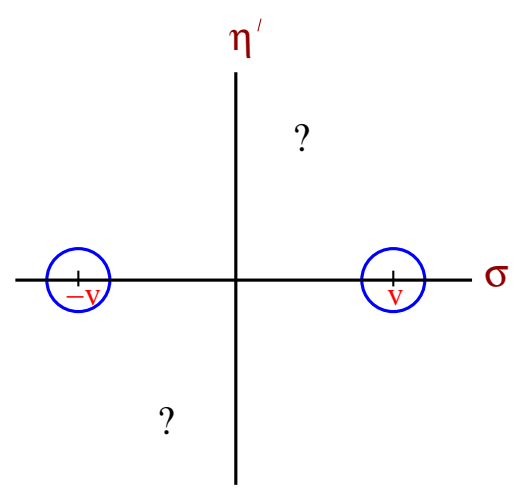

Figure 3: The circles in this figure inclose the two minima in the $\sigma, \eta^{\prime}$ plane located at $\sigma= \pm v$ and $\eta^{\prime}=0$. Can we find any other minima?

on the opposite side of this circle. This technique brings in a modified chiral matrix through the relation $D \gamma_{5}=-\hat{\gamma}_{5} D$ and the winding appears via $\operatorname{Tr} \hat{\gamma}_{5}=2 v$. With Wilson fermions [15] the low lying approximate zero modes are compensated by additional real eigenvalues in the doubler region.

Note that this discussion involves both short and long distances. The zero modes associated with topology are compensated by additional modes lost at the cutoff. This means that it can be dangerous to assume that one can ignore instanton physics by going to short distances. Furthermore it becomes impossible to uniquely separate perturbative and non-perturbative effects; as one changes, say, the scale of the cutoff, small instantons can "fall through the lattice." In general this issue is scheme dependent.

So we conclude that under the transformation of Eq. (4.3), the regulated fermion measure changes by $e^{-i \phi \operatorname{Tr} \gamma_{5}}=e^{-i \phi v}$. This factor changes the weighting of gauge configurations with nonzero winding. Note that this introduces a sign problem for Monte Carlo, but that is not the topic under discussion here.

To end this section, note that the angle $\phi$ I have used here is the conventional $\Theta / N_{f}$. This is since I have given each flavor a common phase. Each contributes equally, and the full trace including flavor space is $\operatorname{Tr} \gamma_{5}=N_{f} v$.

\section{A $Z_{N_{f}}$ symmetry}

I now return to the earlier effective-potential language. I have argued that, because of spontaneous chiral symmetry breaking, there are at least two minima in the $\sigma, \eta^{\prime}$ plane, located as sketched in Fig. 3. Do we know anything about the potential elsewhere in the $\sigma, \eta^{\prime}$ plane? Remarkably the answer is yes; there are actually $N_{f}$ physically equivalent minima in this plane.

At this point it is useful to project out left handed fermion fields

$$
\psi_{L}=\frac{1+\gamma_{5}}{2} \psi
$$

Then, because of the anomaly, a singlet rotation of only the left handed field

$$
\psi_{L} \rightarrow e^{i \phi} \psi_{L}
$$




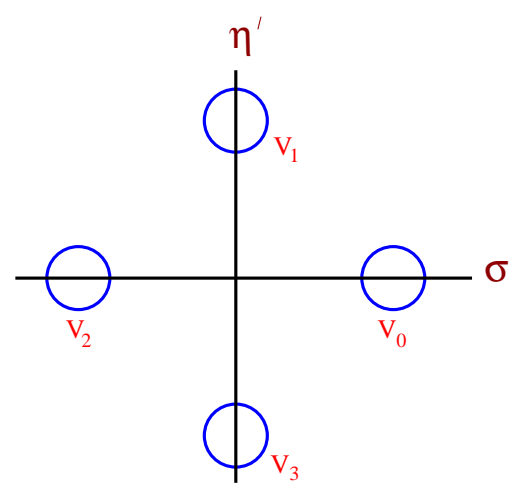

Figure 4: For four flavors, the effective potential has four equivalent minima, marked here with circles, in the $\sigma, \eta^{\prime}$ plane. This generalizes to $N_{f}$ minima with $N_{f}$ flavors.

is not a good symmetry for generic $\phi$. On the other hand, a flavored rotation

$$
\psi_{L} \rightarrow g_{L} \psi_{L}=e^{i \phi_{\alpha} \lambda_{\alpha}} \psi_{L}
$$

is a symmetry for $g_{L} \in S U\left(N_{f}\right)$. The point I wish to emphasize is that for special discrete elements these two types of rotation can cross. In particular I can take $g$ in the center of $S U\left(N_{f}\right)$

$$
g=e^{2 \pi i / N_{f}} \in Z_{N_{f}} \subset S U\left(N_{f}\right),
$$

and we obtain a valid discrete singlet symmetry

$$
\begin{gathered}
\sigma \rightarrow \sigma \cos \left(2 \pi / N_{f}\right)+\eta^{\prime} \sin \left(2 \pi / N_{f}\right) \\
\eta^{\prime} \rightarrow \eta^{\prime} \cos \left(2 \pi / N_{f}\right)-\sigma \sin \left(2 \pi / N_{f}\right) .
\end{gathered}
$$

This $Z_{N_{f}}$ symmetry applies to the effective potential when the quark mass vanishes. Then there are $N_{f}$ equivalent minima in the $\left(\sigma, \eta^{\prime}\right)$ plane. This is sketched for the $N_{f}=4$ case in Fig. 4.

At the chiral Lagrangian level this symmetry arises because $Z_{N}$ is a subgroup of both $S U(N)$ and $U(1)$. At the quark level it can be understood from the fact that the 't Hooft vertex gets a contribution from each flavor and multiplying together the phases $\psi_{L} \rightarrow e^{2 \pi i / N_{f}} \psi_{L}$ from each gives a net factor of unity.

\section{Including the quark mass}

A quark mass term $-m \bar{\psi} \psi \sim-m \sigma$ can be thought of as tilting the effective potential downward in the sigma direction. This picks one vacuum as the lowest. Expanding the potential about the $n$ 'th minimum gives an effective pion mass in the given minimum going as $m_{\pi}^{2} \sim m \cos \left(2 \pi n / N_{f}\right)$. Thus $n=0$ is the true vacuum while the highest minima are unstable in the $\pi_{\alpha}$ direction. Note that multiple truly meta-stable minima become possible when $N_{f}>4$.

While the conventional mass term is proportional to $m \bar{\psi} \psi$, it is interesting to consider a more general term obtained by an anomalous rotation

$$
m \bar{\psi} \psi \rightarrow m \cos (\phi) \bar{\psi} \psi+i m \sin (\phi) \bar{\psi} \gamma_{5} \psi
$$




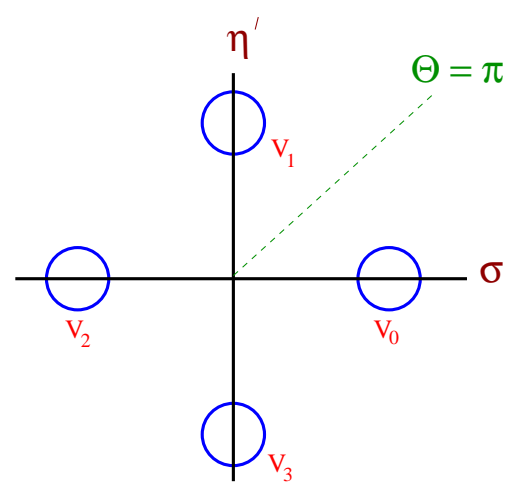

Figure 5: With massive quarks and a twisting angle of $\phi=\pi / N_{f}$, two of the minima in the $\sigma, \eta^{\prime}$ plane become degenerate. This corresponds to a first order transition at $\Theta=\pi$.

This corresponds to tilting the potential downward not in the sigma direction, but in a direction at an the angle $\phi$ in the $\sigma, \eta^{\prime}$ plane. In general this will give an inequivalent theory. For small $\phi$, the vacuum will remain in the vicinity of the minimum at positive $\sigma$; however, as $\phi$ increases through $\pi / N_{f}$, vacuum will jump from this minimum to a neighboring one. This is illustrated in Fig. 5.

In this discussion I have given the mass for each flavor a common phase $\phi$. In more conventional treatments one introduces the sum of these with the definition $\Theta=N_{f} \phi$. The $Z_{N_{f}}$ symmetry implies a $2 \pi$ periodicity in $\Theta$. What has been demonstrated here is that with degenerate light quarks a first order transition is expected at $\Theta=\pi$.

The underlying $Z_{N_{f}}$ can be thought of as a discrete symmetry in mass parameter space

$$
m \rightarrow m \exp \left(\frac{i \pi \gamma_{5}}{N_{f}}\right)
$$

In particular for $N_{f}=4$ a mass term of form $m \bar{\psi} \psi$ is physically equivalent to considering one of form $i m \bar{\psi} \gamma_{5} \psi$. This specific equivalence is only true for $N_{f}$ a multiple of 4 .

\section{Odd $N_{f}$}

At this point it should be beginning to be clear why I had restricted myself to even $N_{f}$. Now consider an odd number of flavors, $N_{f}=2 N+1$. The crucial point is that -1 is not an element of $S U(2 N+1)$. This means that $m>0$ and $m<0$ not equivalent! In particular a negative mass represents $\Theta=\pi$ and will exhibit spontaneous $\mathrm{CP}$ violation with $\left\langle\eta^{\prime}\right\rangle \neq 0$. Fig. 6 sketches the situation for $S U(3)$.

The fact that the sign of the quark mass is relevant for an odd number of flavors is something not seen in perturbation theory. In any given Feynman diagram, the sign of the mass can be flipped by a $\gamma_{5}$ rotation. Thus positive and negative mass three flavor QCD have identical perturbative expansions and yet are physically different. This is a simple example of the remarkable fact that inequivalent theories can have identical perturbative expansions!

A special case of an odd number of flavors is one-flavor QCD. In this situation the anomaly removes all chiral symmetry and there is a unique minimum in the $\sigma, \eta^{\prime}$ plane, as sketched in Fig. 7. This minimum does not occur at the origin, being shifted to $\langle\bar{\psi} \psi\rangle>0$ by the 't Hooft 


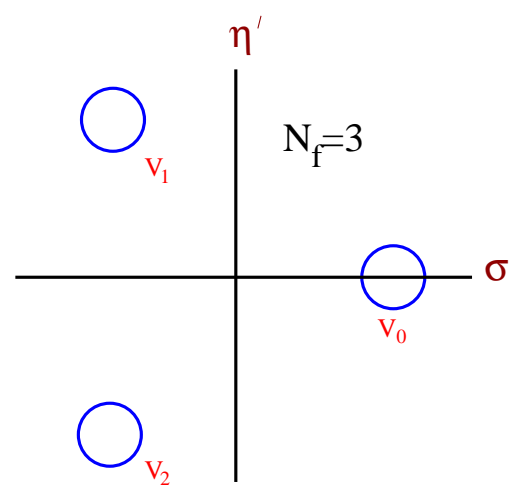

Figure 6: For odd $N_{f}$, such as the $S U(3)$ case sketched here, QCD is not symmetric under changing the sign of the quark mass. Negative mass corresponds to taking $\Theta=\pi$.

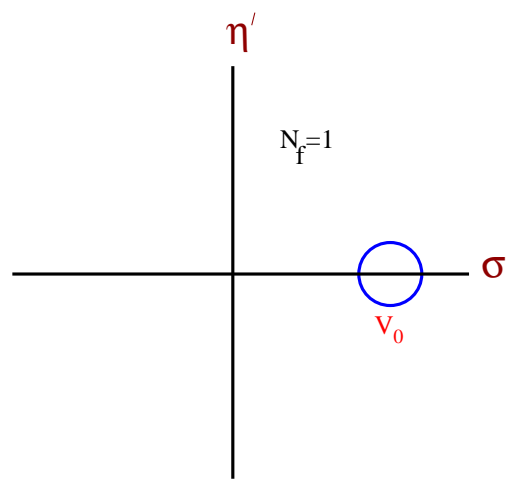

Figure 7: The effective potential for one-flavor QCD with small quark mass has a unique minimum in the $\sigma, \eta^{\prime}$ plane. The minimum is shifted from zero due to the effect of the 't Hooft vertex.

vertex, which for one flavor is just an additive mass shift [16]. Unlike the case with more flavors, this expectation cannot be regarded as a spontaneous symmetry breaking since there is no chiral symmetry to break. Any regulator that preserves a remnant of chiral symmetry in the one flavor theory must inevitably fail [9]. Note also that there is no longer the necessity of a first order phase transition at $\Theta=\pi$. It has been argued [17] that for finite quark mass such a transition will occur if the mass is sufficiently negative, but physics is analytic in $m$ in a finite region around vanishing mass.

It is important to remember that the details of the instanton effects are scheme dependent; this is sometimes called the "renormalon" ambiguity [18]. For the one flavor case this means the usual polar coordinates $(m, \Theta)$ are singular. Indeed, it is more natural to use $(\operatorname{Re} m, \operatorname{Im} m)$ as our fundamental parameters. The ambiguity is tied to rough gauge configurations of ill-defined winding number. Even the overlap operator does not solve this issue since it is not unique, depending on a parameter often called the "domain wall height." Because of this, $m=0$ for a non-degenerate quark is an ambiguous concept. In Appendix A I discuss this ambiguity from the point of view of the renormalization group. 


\section{When is rooting okay?}

Starting with four flavors, can one adjust $N_{f}$ down to one using the formal expression

$$
\left|\begin{array}{cccc}
D+m & 0 & 0 & 0 \\
0 & D+m & 0 & 0 \\
0 & 0 & D+m & 0 \\
0 & 0 & 0 & D+m
\end{array}\right|^{\frac{1}{4}}=|D+m| ?
$$

This has been proposed and is widely used as a method for eliminating the extra species appearing with staggered fermion simulations.

It is important to emphasize that asking about the viability of Eq. 8.1 is a vacuous question outside the context of a regulator. Field theory has divergences that need to be controlled, and, as we have seen above, the appearance of anomalies requires care. In particular, the regulated theory must break all anomalous symmetries.

So we must apply Eq. 8.1 before removing the regulator. This is generally expected to be okay as long as the regulator breaks any anomalous symmetries appropriately on each of the four factors. For example, we expect rooting to be valid for four copies of the overlap operator. This satisfies a modified chiral symmetry $D \gamma_{5}=-\hat{\gamma}_{5} D$ where the gauge winding $v$ appears in the gauge dependent matrix $\hat{\gamma}$ through $\operatorname{Tr} \hat{\gamma}_{5}=2 v$.

But now suppose we try to force the $Z_{4}$ symmetry in mass parameter space before we root. This is easily done by considering the determinant

$$
\left|\begin{array}{cccc}
D+m e^{\frac{i \pi \gamma_{5}}{4}} & 0 & 0 & 0 \\
0 & D+m e^{\frac{-i \pi \gamma_{5}}{4}} & 0 & 0 \\
0 & 0 & D+m e^{\frac{3 i \pi \gamma_{5}}{4}} & 0 \\
0 & 0 & 0 & D+m e^{\frac{-3 i \pi \gamma_{5}}{4}}
\end{array}\right| .
$$

This maintains the $m \rightarrow m e^{i \pi \gamma_{5} / 2}$ symmetry through a permutation of the four flavors. This still gives a valid formulation of the four flavor theory at vanishing $\Theta$ because the imposed phases cancel. But expressed in this way, we start with four one-flavor theories with different values of $\Theta$. Were we to root this form, we would be averaging over four inequivalent theories. This is not expected to be correct, much as we would not expect rooting two different masses to give a theory of the average mass; i.e.

$$
\left(\left|D+m_{1}\right|\left|D+m_{2}\right|\right)^{1 / 2} \neq\left|D+\sqrt{m_{1} m_{2}}\right| .
$$

So we have both a correct and an incorrect way to root a four flavor theory down to one. What is the situation with staggered fermions, the primary place where rooting has been applied? The problem is that the kinetic term of the staggered action maintains one exact chiral symmetry. Without rooting this is an allowed symmetry amongst what are usually called "tastes." Under this symmetry there are two tastes of each chirality. But, because of this exact symmetry, which contains a $Z_{4}$ subgroup, rooting to reduce the theory to one flavor is not expected to be valid. In particular, rooting does not remove the $Z_{4}$ discrete symmetry in the mass parameter, a symmetry which is anomalous in the one flavor theory. Thus, as in the above example, the tastes are not equivalent and rooting averages inequivalent theories. 
The conclusion is that rooted staggered fermions are not QCD. So, what is expected to go wrong? The unbroken $Z_{4}$ symmetry will give rise to extra minima in the effective potential as a function of $\sigma$ and $\eta^{\prime}$. Forcing these minima would most likely drive the $\eta^{\prime}$ mass down from its physical value. This shift should be rather large, of order $\Lambda_{Q C D}$. This is testable, but being dominated by disconnected diagrams, may be rather difficult to verify in practice.

\section{Summary}

We have seen that QCD with $N_{f}$ massless flavors has a discrete flavor-singlet $Z_{N_{f}}$ chiral symmetry. Associated with this is a first order transition at $\Theta=\pi$ when $m \neq 0$. As a consequence, the sign of the mass is significant for $N_{f}$ odd, a property not seen in perturbation theory. Going down to the $N_{f}=1$ case, no chiral symmetry survives, leaving $m=0$ unprotected from additive renormalization. And finally, this structure is inconsistent with rooted staggered quarks due to an anomalous $Z_{4}$ symmetry being improperly preserved.

\section{Appendix A: The renormalization group and the quark mass}

The ambiguity in defining the mass of a non-degenerate quark can be nicely formulated in the renormalization group framework [8]. The renormalization group equation for the bare quark mass

$$
a \frac{d m}{d a}=m \gamma(g)=m\left(\gamma_{0} g^{2}+\gamma_{1} g^{4}+\ldots\right)+\text { non-perturbative }
$$

can in general contain a non-perturbative part that vanishes faster in $g$ than any power. From the perturbative part and using the corresponding flow equation for the bare coupling $g$, we learn that the bare quark mass runs to zero logarithmically with the cutoff

$$
m \propto g^{\gamma_{0} / \beta_{0}}\left(1+O\left(g^{2}\right)\right) \rightarrow_{a \rightarrow 0} 0 .
$$

where $g$ is the bare gauge coupling, which, by asymptotic freedom, runs to zero. We can thus define a renormalized quark mass

$$
m_{r}=\lim _{a \rightarrow 0} m g^{-\gamma_{0} / \beta_{0}}
$$

In general the numerical value of $m_{r}$ depends on the details of the regularization scheme used. The anomaly, through the 't Hooft vertex, contributes a non-perturbative part $\sim m^{N_{f}-1}$ to the mass flow. For the case of $N_{f}=1$, this ceases to vanish in the massless limit. Indeed, remembering that

$$
m_{\eta^{\prime}} \propto \frac{1}{a} e^{-1 / 2 \beta_{0} g^{2}} g^{-\beta_{1} / \beta_{0}^{2}}
$$

we might expect a similar form to appear in renormalization group equation for the mass. This is particularly so if the $\eta^{\prime}$ mass is used as a physical observable defining the renormalization scheme. Note that this non-perturbative expression formally diverges if we take $a$ to zero without the appropriate simultaneous decrease of the coupling. Allowing such a term can give rise to an additive shift in the renormalized quark mass. As an extreme example, consider a new scheme defined by

$$
\begin{gathered}
\tilde{a}=a \\
\tilde{g}=g \\
\tilde{m}=m-m_{r} g^{\gamma_{0} / \beta_{0}} \times \frac{e^{-1 / 2 \beta_{0} g^{2}} g^{-\beta_{1} / \beta_{0}^{2}}}{\Lambda a} .
\end{gathered}
$$


This is crafted so that on the renormalization group trajectory the last factor approaches unity. With this particular non-perturbative redefinition of parameters we have

$$
\tilde{m}_{r} \equiv \lim _{a \rightarrow 0} \tilde{m} \tilde{g}^{-\gamma_{0} / \beta_{0}}=m_{r}-m_{r}=0 .
$$

Thus in the one flavor theory it is always possible to find a scheme where the renormalized quark mass vanishes! We conclude that $m=0$ for a non-degenerate quark is an ambiguous concept. Of course, with degenerate quarks $m_{\pi}=0$ defines $m=0$.

\section{References}

[1] E. Vicari and H. Panagopoulos, arXiv:0803.1593 [hep-th].

[2] R. F. Dashen, Phys. Rev. D 3, 1879 (1971).

[3] G. 't Hooft, Phys. Rev. D 14, 3432 (1976) [Erratum-ibid. D 18, 2199 (1978)].

[4] E. Witten, Annals Phys. 128, 363 (1980).

[5] C. Rosenzweig, J. Schechter and C. G. Trahern, Phys. Rev. D 21, 3388 (1980);

R. L. Arnowitt and P. Nath, Nucl. Phys. B 209, 234 (1982);

P. Nath and R. L. Arnowitt, Nucl. Phys. B 209, 251 (1982);

K. Kawarabayashi and N. Ohta, Nucl. Phys. B 175, 477 (1980);

K. Kawarabayashi and N. Ohta, Prog. Theor. Phys. 66, 1789 (1981);

N. Ohta, Prog. Theor. Phys. 66, 1408 (1981) [Erratum-ibid. 67, 993 (1982)].

[6] D. Boer and J. K. Boomsma, Phys. Rev. D 78, 054027 (2008) [arXiv:0806.1669 [hep-ph]].

[7] M. Creutz, Phys. Rev. D 52, 2951 (1995) [arXiv:hep-th/9505112].

[8] M. Creutz, Phys. Rev. Lett. 92, 162003 (2004) [arXiv:hep-ph/0312225].

[9] M. Creutz, arXiv:0810.4526 [hep-lat]; also see references therein.

[10] M. Creutz, Annals Phys. 324, 1573 (2009) [arXiv:0901.0150 [hep-ph]].

[11] A. V. Smilga, Phys. Rev. D 59, 114021 (1999) [arXiv:hep-ph/9805214].

[12] M. H. G. Tytgat, Phys. Rev. D 61, 114009 (2000) [arXiv:hep-ph/9909532].

[13] K. Fujikawa, Phys. Rev. Lett. 42, 1195 (1979);

K. Fujikawa, Phys. Rev. D 21, 2848 (1980) [Erratum-ibid. D 22, 1499 (1980)].

[14] P. H. Ginsparg and K. G. Wilson, Phys. Rev. D 25, 2649 (1982);

H. Neuberger, Phys. Lett. B 417 (1998) 141 [arXiv:hep-lat/9707022].

[15] K. G. Wilson, in New Phenomena In Subnuclear Physics. Part A. Proceedings of the First Half of the 1975 International School of Subnuclear Physics, Erice, Sicily, July 11 - August 1, 1975, ed.

A. Zichichi, Plenum Press, New York, 1977, p. 69.

[16] M. Creutz, Annals Phys. 323, 2349 (2008) [arXiv:0711.2640 [hep-ph]].

[17] M. Creutz, Annals Phys. 322, 1518 (2007) [arXiv:hep-th/0609187].

[18] I. I. Y. Bigi, M. A. Shifman, N. G. Uraltsev and A. I. Vainshtein, Phys. Rev. D 50, 2234 (1994) [arXiv:hep-ph/9402360]. 